CNS Spectrums (2017), 22, 282-289. C Cambridge University Press 2016. The online version of this article is published within an Open Access environment subject to the conditions of the Creative Commons Attribution-NonCommercial-ShareAlike licence <http://creativecommons.org/licenses/by-nc-sa/4.0/>. The written permission of Cambridge University Press must be obtained for commercial re-use.

doi:10.1017/S1092852916000286

\title{
The medial forebrain bundle as a target for deep brain stimulation for obsessive-compulsive disorder
}

\author{
Volker A. Coenen, ${ }^{1 *}$ Thomas E. Schlaepfer, ${ }^{2}$ Peter Goll, ${ }^{3}$ Peter C. Reinacher, ${ }^{1}$ \\ Ulrich Voderholzer, ${ }^{4}$ Ludger Tebartz van Elst, ${ }^{3}$ Horst Urbach, ${ }^{5}$ and Tobias Freyer ${ }^{3}$
}

\footnotetext{
${ }^{1}$ Department of Stereotactic and Functional Neurosurgery, Freiburg University Medical Center, Germany

2 Department of Psychiatry and Psychotherapy, University Hospital, Bonn, Germany

${ }^{3}$ Department of Psychiatry and Psychotherapy, Freiburg University Medical Center, Freiburg, Germany

${ }^{4}$ Schön Klinik Roseneck, Prien am Chiemsee, Germany

${ }^{5}$ Department of Neuroradiology, Freiburg University Medical Center, Freiburg, Germany
}

Deep brain stimulation (DBS) is a promising putative modality for the treatment of refractory psychiatric disorders such as major depression and obsessive-compulsive disorder (OCD). Several targets have been posited; however, a clear consensus on differential efficacy and possible modes of action remain unclear. DBS to the supero-lateral branch of the medial forebrain bundle (slMFB) has recently been introduced for major depression (MD). Due to our experience with slMFB stimulation for MD, and because OCD might be related to similar dysfunctions of the reward system, treatment with slMFB DBS seams meaningful. Here we describe our first 2 cases together with a hypothetical mode of action.

We describe diffusion tensor imaging (DTI) fiber tractographically (FT)-assisted implantation of the bilateral DBS systems in 2 male patients. In a selected literature overview, we discuss the possible mode of action. Both patients were successfully implanted and stimulated. The follow-up time was 12 months. One patient showed a significant response (Yale-Brown Obsessive-Compulsive Scale [YBOCS] reduction by 35\%); the other patient reached remission criteria 3 months after surgery (YBOCS $<14$ ) and showed mild OCD just above the remission criterion at 12 months follow-up.

While the hypermetabolism theory for OCD involves the cortico-striato-thalamo-cortical (CSTC) network, we think that there is clinical evidence that the reward system plays a crucial role. Our findings suggest an important role of this network in mechanisms of disease development and recovery. In this uncontrolled case series, continuous bilateral DBS to the sIMFB led to clinically significant improvements of ratings of OCD severity. Ongoing research focuses on the role of the reward system in $\mathrm{OCD}$, and its yet-underestimated role in this underlying neurobiology of the disease.

Received 7 March 2016; Accepted 16 April 2016; First published online 8 June 2016

Key words: Deep brain stimulation (DBS), depression, functional neurosurgery, obsessive-compulsive disorder (OCD), medial forebrain bundle (MFB), Yale-Brown Obsessive Compulsive Scale (YBOCS).

\section{Introduction}

Over the last 15 years, deep brain stimulation (DBS) has been demonstrated to be a promising putative therapy for patients with treatment-refractory obsessive-compulsive disorder (OCD). This is because of the procedure's reversibility and adjustability, and its comparable efficacy to ablative surgeries that were used previously. ${ }^{1,2}$ Several meta-analyses and reviews summarize the potential of DBS in OCD; however, the mechanisms underlying its

* Address for correspondence: Volker A. Coenen, M.D., Department of Stereotactic and Functional Neurosurgery, Freiburg University Medical Center, Breisacher Str. 64, 79106 Freiburg (i.Br.), Germany. (Email: volker.coenen@uniklinik-freiburg.de) therapeutic effects remain unclear. $^{3,4}$ Targets under investigation are the anterior limb of the internal capsule (ALIC; often also ventral capsule $=\mathrm{vc}$ ), the ventral stiatum (vs), the medial subthalamic nucleus (mSTN), the inferior thalamic peduncle, and the nucleus accumbens (NAC). ${ }^{5}$ These structures have been selected because they have been considered as genuine parts of the OCD circuitry, ${ }^{6}$ and they have previously been used as stereotactic lesions targets or were correlated with improvements of comorbid OCD symptoms in patients undergoing DBS surgery for other indications (eg, the STN in Parkinson disease). Recently, convincing antidepressant effects of stimulation of the supero-lateral branch of the medial forebrain bundle (slMFB) in severely depressed 
patients (ie, those with major depressive disorder [MDD]) have been reported. ${ }^{7}$ It was hypothesized that the possible, more rapid onset of clinical improvement, the possible low rate of side-effects, and the low current needed were all due to the more direct stimulation of the crucial white matter tract connecting the structures involved in the pathology of depression, namely the medial forebrain bundle (MFB). ${ }^{8-10}$ Moreover, some of the established target regions for DBS in OCD are interconnected through the MFB, ${ }^{8,10,11}$ supporting the notion that direct stimulation of the reward system (and its main regulating structure, the MFB) could-as in depression-result in strong clinical effects in OCD patients. Here, we present 2 cases with treatment-refractory OCD with a significant and sustained therapeutic benefit after DBS of the slMFB. We also discuss a mode of action that might explain the proposed efficacy of this approach in the context of reward system modulation.

\section{Materials and Methods}

DBS of the anterior limb of the internal capsule (ventral capsule) and the ventral striatum (vc/vs) for therapy refractory OCD is an established therapy in Europe and has received a (preliminary) CE-mark. The use of the slMFB as target for DBS is a compassionate use along this line of argumentation and was brought to the attention of the local ethics committee of Freiburg University before the implantations in both cases. The patients gave written informed consent especially with respect to the experimental nature of the treatment.

\section{Imaging and tractographic approach}

Anatomical and diffusion tensor imaging was performed on a clinical 3 Tesla MRI system (Siemens Magnetom Trio Tim System 3T, Erlangen, Germany) a few days before surgery under mild sedation. Specifications were as follows: anatomical sequences: 12-channel head coil, 3D magnetization prepared rapid gradient echo (MPRAGE): TR $1390 \mathrm{~ms}$, TE $2.15 \mathrm{~ms}$, TI $800 \mathrm{~ms}$, flip angle $15^{\circ}$, voxel size $1.0 \times 1.0 \times 1.0 \mathrm{~mm}^{3}$, acquisition time 3:15 min; 3D T2 SPACE-sequence: TR $2500 \mathrm{~ms}$, TE $231 \mathrm{~ms}$, echo train length 141, flip angle variable, voxel size $1.0 \times 1.0 \times 1.0 \mathrm{~mm}^{3}$, acquisition time $6: 42 \mathrm{~min}$; diffusion tensor imaging: single shot 2D SE EPI, TR $10000 \mathrm{~ms}$, TE $94 \mathrm{~ms}$, diffusion values $\mathrm{b}=0 \mathrm{~s} / \mathrm{mm}^{2}$, $\mathrm{b}=1000 \mathrm{~s} / \mathrm{mm}^{2}$, diffusions directions 61 , slice count 69 , voxel size $2.0 \times 2.0 \times 2.0 \mathrm{~mm}^{3}$, acquisition time 11:40 min. Deformation correction of the EPI sequence was performed according to Zaitsev et al. ${ }^{12}$

\section{Fiber tracking}

Deterministic FT was performed on a Linux workstation using StealthViz DTI (Medtronic Navigation, Louisville,
CO, USA). Rendition of the individual slMFB with deterministic tractography was performed as described before with a single region of interest. ${ }^{7,10}$

\section{Surgery}

Stereotactic implantation of bilateral DBS electrodes was performed under mild analgesia with the patient responsive using a Leksell G-Frame (Elekta, Sweden) using typical coronal entry points. The DTI tractographic rendition of the slMFB served to determine the individual target regions (inferior borders of slMFB). Microelectrode recording on 3 trajectories (anterior, lateral, and central) was applied to electrophysiologically verify the corridor between the red nucleus (posteromedially) and the subthalamic region (laterally) and more inferior the substantia nigra (laterally, see Figure 1). After a macrostimulation test to search for antiaversive (anti-OCD) effects of stimulation and the oculomotor threshold, the final DBS electrodes (model 3389, Medtronic) were implanted under lateral x-ray control. In a second step and under general anesthesia, the DBS electrodes were subcutaneously connected to an internal pulse generator that was located abdominally (ACTIVA PC, Medtronic).

\section{Case descriptions}

\section{Patient 1}

Patient 1 was a 32 -year-old man who had suffered from OCD since the age of 10 . His family first sought professional help when the patient was 13 . Since then he had been treated by several psychiatrists and psychotherapists, including cognitive behavioral therapy with exposure and response prevention (CBT with ERP) and numerous medications administered at adequate dosages and for adequate durations (clomipramine, SSRIs, augmentation with second-generation antipsychotics). Neither inpatient nor outpatient treatment had a substantial effect on the course of his OCD, with symptoms steadily deteriorating over time. Despite the rather slow but successful completion of training as a baker and as an office administrator, he was never able to work in these professions due to the severity of his OCD. At the time of assessment, he had been receiving invalidity pension for 4 years and had significant functional and social impairment. Baseline evaluation revealed severe contamination obsessions with associated washing compulsions and pronounced avoidance behavior. He was living in his parents' house, but he inhabited a "clean part" in which no other family member was allowed to set foot. He avoided shared facilities within or outside the house to the greatest possible extent. Visits of his brother, who was living in a distant city, turned out to be a reliable trigger of 

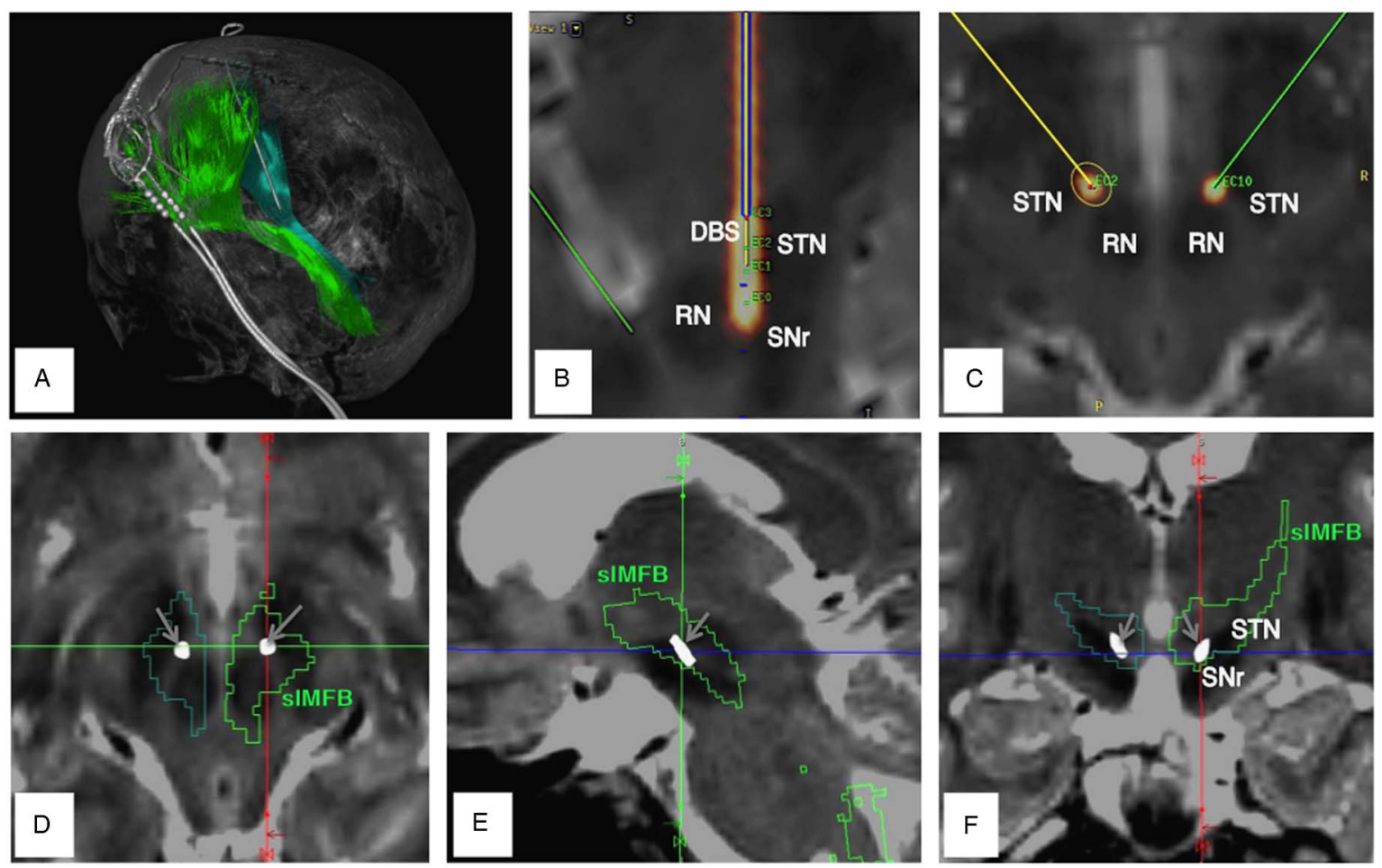

FIGURE 1. Representation of DTI tractographically assisted sIMFB DBS in patient 1. (A) Three-dimensional rendition of sIMFB (light green, left; dark green,

right) with DBS electrodes (white), as seen from posterior and left. (B, C) Superimposition of computed tomography (with electrode artifact = DBS, orange) and MRI (T2). The DBS electrode is situated in a narrow corridor between the red nucleus (RN, medial) and the subthalamic nucleus (STN)/substantia nigra (SNr) region, lateral. This is the typical position. (D, E, F) Axial, sagittal, and coronal views, respectively, of electrode implantation sites with sIMFB outlines (from DTI tractography). Gray arrows indicate DBS electrode artifacts (white dots).

substantial OCD crises due to the patient's obsessional association of big cities and infectious particles. At baseline assessment, he was medicated with $20 \mathrm{mg}$ escitalopram and $10 \mathrm{mg}$ zolpidem. At this time, his YBOCS score was 39, his Montgomery-Åsberg Depression Rating Scale (MADRS) score was 21, and his global assessment of functioning (GAF) score was 30 (see Figure 2).

\section{Patient $2^{\dagger}$}

Our second subject was a 51-year-old man who showed a similar course of disease with initial manifestation of OCD symptoms at the age of 15 . His first contact with healthcare professionals was not until his late $20 \mathrm{~s}$ though. Since then, he had received extensive therapies, including CBT with ERP and numerous medications simultaneously or separately administered at adequate dosages and for adequate durations (clomipramine, SSRIs, augmentation with second-generation antipsychotics). Neither inpatient nor outpatient interventions had any substantial effect on the course of his OCD,

\footnotetext{
${ }^{\dagger}$ Patient 2 unexpectedly died 21 months after DBS surgery and stimulation. The official cause (death certificate) was "death by natural causes". However, a postmortem was not performed. The death occurred after the acceptance of this paper.
}

which worsened progressively. The deteriorating course of disease affected his professional career significantly. After working a few years in his trained job as a painter, he eventually ended up at the public cleansing service. At this post, he was frequently asked to remove animal cadavers, triggering even more extreme fears of contamination and leading to his occupational invalidity in 2002. At the time of assessment, he displayed significant functional and social impairment. The most prominent features were severe violent obsessions with associated controlling compulsions and pronounced avoidance behavior. Thus, he had been barely able to attend a sheltered workshop. Eventually he withdrew from any social activities except schematic Sunday outings with his mother, even though he received extensive inpatient treatment during that time. At baseline assessment, he was medicated with $150 \mathrm{mg}$ clomipramine, $1075 \mathrm{mg}$ lithium, and $50 \mathrm{mg}$ clozapine. His YBOCS score was 30 , his Beck Depression Inventory (BDI) score was 36, and his GAF score was 40 (see Figure 2).

\section{Results}

Intraoperatively, we detected some decrease of the aversiveness of predefined supposedly contaminating 


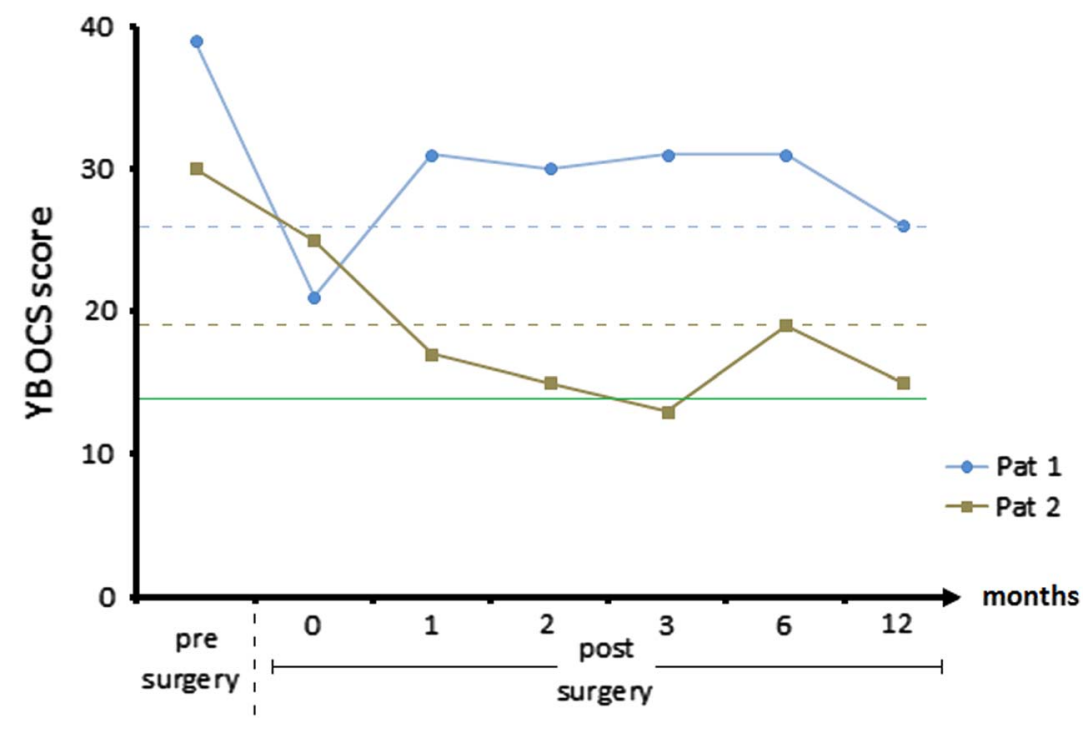

FIGURE 2. Yale-Brown Obsessive-Compulsive Scale (YBOCS) scores before surgery and 1 week, 1 month, 2 months, 3 months, 6 months, and 1 year after implantation. The dotted lines represent the individual thresholds of response (defined by YBOCS reduction of $35 \%{ }^{30}$ ), and the green line represents remission (defined by YBOCS $<14^{30}$ ).

stimuli/objects (patient 1 was exposed to T. Freyer's shoe, positioned on the patient's chest; patient 2 as exposed to a photo of the wheel of a service cart from the psychiatric ward). Typical heart rate increase was seen during acute stimulation, as described in the initial series of depressed patients. ${ }^{7}$ We detected typical oculomotor effects at the deepest point of the implantation in both cases. There was no worsening of the neural status. No intracranial bleeding or infection occurred. Both patients had uneventful postoperative courses. The patients underwent postoperative helical CT at day of surgery in order to determine the achieved electrode positions (by image fusion to the planning data). Both patients showed electrode positions in the center of the slMFB as planned without any bleeding (Figure 1 exemplarily shows patient 1 ). Effective contacts (center of 2 cathodal contacts) were expressed in mid-commissural point (MCP) system coordinates: Patient 1: $x=7.6$; $\mathrm{y}=-1.2, \mathrm{z}=-2.5$ (right); $\mathrm{x}=7.6, \mathrm{y}=-1.2, \mathrm{z}=-2.7$ (left). Patient 2: $\mathrm{x}=9, \mathrm{y}=-2.1, \mathrm{z}=-2.4$ (right); $\mathrm{x}=6.2$, $\mathrm{y}=-2.1 ; \mathrm{z}=-4.4$ (left). Negative $\mathrm{z}$-value indicates position below the MCP.

Stimulation was initiated after several days, and both patients showed some double vision during the titration of the target current, which was overcome by reprogramming as described before. ${ }^{7}$ No other adverse events were detected.

\section{Stimulation parameters}

Continuous bilateral stimulation was performed in a bipolar stimulation setting that was titrated to a minimal target current (2.5 milliampere $[\mathrm{mA}])$ as described before $^{7}$ and adjusted just below the threshold for oculomotor side effects. In both patients, the second most inferior electrode contact served as anode, and the 2 electrode contacts above served as cathodes. This is the typical stimulation pattern for this target region. ${ }^{7}$ All stimulations were performed with a frequency of $130 \mathrm{~Hz}$ and a pulse width of $60 \mu \mathrm{s}$. In patient 1 , amplitudes were $3.6 \mathrm{~mA}$ (left) and $3.5 \mathrm{~mA}$ (right). In patient 2, amplitudes were $2.5 \mathrm{~mA}$ (left) and 2.7 (right).

Patients were discharged after approximately 1 week and were further seen on a regular basis as outpatients by our interdisciplinary team of psychiatrists and neurosurgeons. Over time, according to disease symptoms, gradual adjustments were made in stimulation amplitudes. The above mentioned parameters are the resulting settings that yielded the best patient specific outcome.

\section{Disease specific outcomes}

\section{Patient 1}

Patient 1 showed a strong immediate effect of surgery in terms of affect regulation and compulsive behavior. Though he never presented symptoms of hypomania, there was a significant change in his beforehand "troubled" appearance. He reported less worrying and less attention bias toward OCD-relevant stimuli. This resulted in much more relaxed behavior on the ward, less compulsive checking, and less reassurance behavior. This effect vanished within 7 days after surgery; the first YBOCS rating after 4 weeks revealed a symptom reduction that did not reach response criteria. Within the following months, however, his condition further stabilized. He was able to move more freely within his parents' home and to accept 
visits from his brother, even to touch him. He gained confidence in reengaging in former hobbies and in starting outpatient treatment, which has been impossible pre-surgery due to a necessary 30-minutes car drive and insufferable avoidance because of "pseudotraumatic" experiences in former therapy. He recommenced therapy 14 months after surgery (the interval was due to waiting time). At 1-year follow-up, his YBOCS reduction exceeded $35 \%$ (YBOCS score was 30 presurgery, and 26 at 12 months follow-up), and his BDI revealed mild depressive symptoms with a score of 16 .

\section{Patient 2}

Patient 2 displayed improved affect regulation and less compulsive behavior within hours after surgery; these sustained until transference to our psychiatric ward. Within 1 week after surgery, confrontational therapeutic interventions of unexpected intensity could be performed with a strikingly less anxious patient. During the course of therapy, he found alternative strategies for situations in which he reassured himself in contact with medical personell and progressed to his top-level confrontational situation-a rat trap containing poison and a warning sign. After 4 weeks, he was able to transfer his newly gained flexibility to his private life, started library visits, and decided to take up his former occupational activities with a positive spirit. Even beyond 1-year follow-up, he succeeded in implementing his intended goals without being held back by less frequent avoidance and control behaviors. Within that time period, his YBOCS results scratched a $50 \%$ reduction level and remained stable within a corridor exceeding $30 \%$ reduction consistent with clinical observations (YBOCS score was 30 presurgery, and 15 at 12 months follow-up).

\section{Discussion}

The symptoms of OCD are thought to be related to a dysregulation in the cortico-striato-thalamo-cortical circuit. ${ }^{6,13-15}$ In a mouse model, a behavior reminiscent of OCD-type behavior (grooming) was elicited through repeated optogenetic stimulation (activation over days) of the ventromedial striatum (VMS) and orbitofrontal cortex (OFG). ${ }^{13}$ Thus it is the long-term and not the short-term activation that presumably leads to typical OCD symptoms. Lesion surgery in the anterior limb of the internal capsule (ALIC) as well as ALIC DBS (more recently coined ventral capsule ventral striatum [vc/vs] DBS) typically reduces the high metabolic activity of the VMS and OFC. ${ }^{15}$ Several mechanisms might explain this: As a proposed net effect, high activity in the orbitofrontal cortex (OFG)/prefrontal cortex (PFC) circuit needs to be reduced to be therapeutically effective. Optogenetic research in rodents suggests that as a consequence of high-frequency phasic activity in the ventral tegmental area (VTA) dopaminergic neurons (and their cortical pendants in the PFC), animals react with increased susceptibility to stress (the behavioral hallmark to depression and OCD). A (functional) disconnection of the VTA and PFC ameliorates this effect. ${ }^{16}$

Clinically, lesions of the anterior limb of internal capsule will simultaneously disconnect both the bilateral anterior thalamic radiation (ATR) and the MFB system, ${ }^{10,11,17,18}$ which are in close proximity to ALIC and converge onto the PFC (Figure 3). Hurwitz et al ${ }^{17}$ showed that 2 fiber pathways degenerate in the anterior limb of the internal capsule after successful lesion surgery. ${ }^{17}$ High activity in the ATR (which directly connects the PFC and DMT) is in agreement with the theory of CSTC hypermetabolism. Very likely, this is the second mechanism that reduces the high activity of the PFC, namely disconnection of the VTA-PFC fibers in anterior capsule (cf. ALIC) lesions. The fact that a significant number of patients show fatigue after anterior capsule lesions supports this notion, because it is likely related to the disconnection of the ascending fibers of the reward system (MFB) on their way to the PFC. ${ }^{17,18}$ In our 2 patients, we have seen a "stun" effect, with significantly decreased anxiety and decreased social retreat (but without fatigue) in the days after surgery and before stimulation. A functional disconnection of a highly active VTA from the PFC with an anti-OCD effect might explain this observation of a transient lesion due to electrode implantation. The authors have likewise seen this stun effect in patients with major depression and slMFB DBS.

ALIC DBS leads to a reduced activity in the dorsomedial thalamus (DMT; Figure 3) and significantly in the OFC as part of the ATR system. ${ }^{15}$ This cannot readily be explained by a stimulation of ATR fibers. However, according to our observations, ALIC DBS may exert its effects through activation of the distal slMFB in $\mathrm{ALIC}^{10,11}$ (Figure 3). This would lead to a down-regulation of the OFC and indirectly to a downregulation of the ATR system (Figure 4).

According to this heuristic, OCD patients would initiate rituals (checking, hand washing, etc) because they transiently activate their reward circuitry with a synchronous tonic activity of VTA dopaminergic neurons and a reduction of high-frequency oscillations (the rituals are expected to be rewarding/anxiolytic). This might well reduce susceptibility to stress and at the same time decrease anxiety levels. These neurons only transiently respond and swing back into high oscillatory activity (Figure 4).

Although the mode of action of sIMFB DBS remains speculative at this time, this permits us to extrapolate to a plausible mechanism: As a careful translation from rodent research, ${ }^{9}$ we have previously suggested (for depression) a modulating effect that does not directly exert its effects over the ascending slMFB fibers but merely over a 


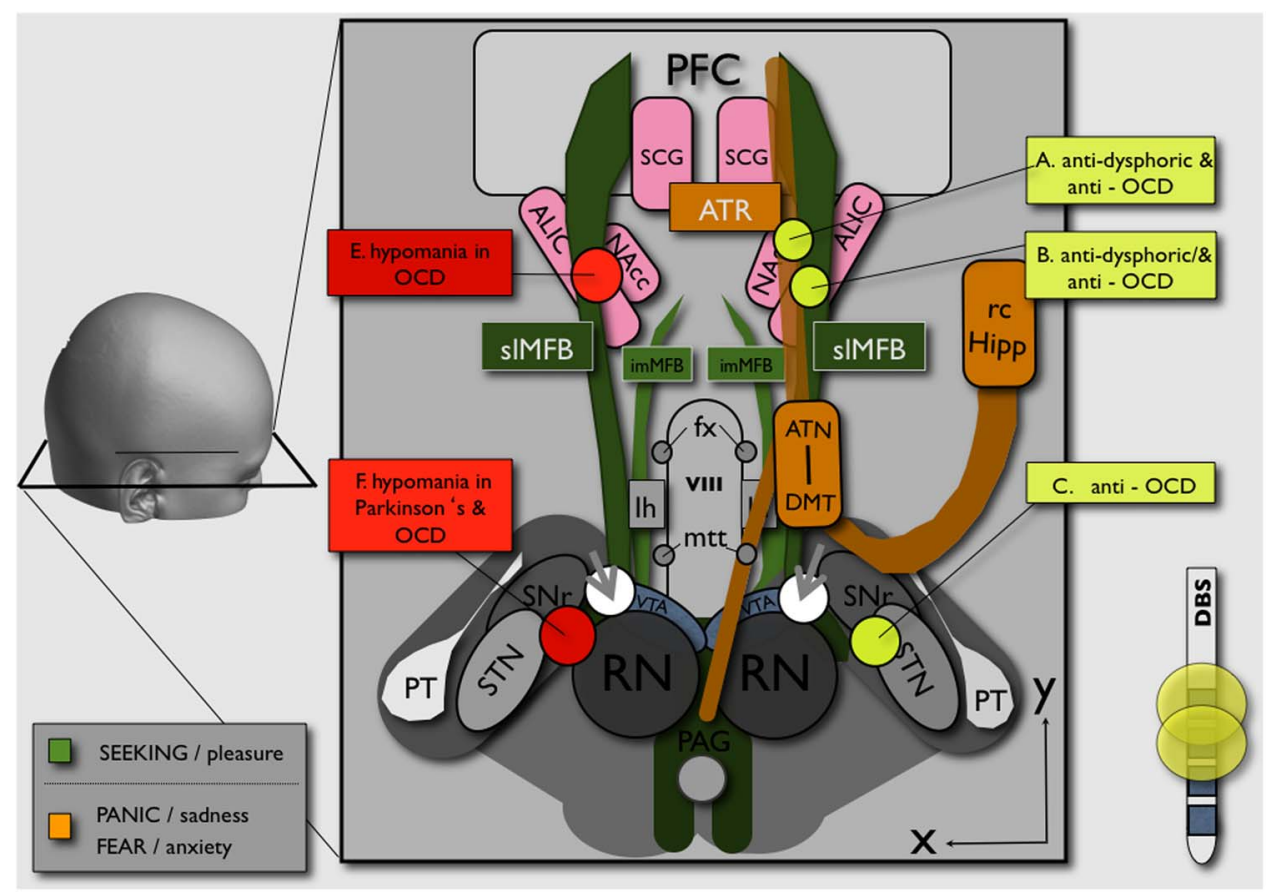

FIGURE 3. Schematic overview of an alternative and putative OCD circuitry. The SEEKING system (anatomically confluent with the MFB, promoting reward) is represented in green. The FEAR system (anatomically realized as the ATR, promoting anxiety if highly active) is intimately connected with the reward system (for better overview, only shown on the right). White spheres (gray arrowheads) indicate sIMFB stimulation site lateral to the VTA. Yellow spheres (only shown on the right) indicate clinically effective sites for OCD treatment (DBS or lesion). ${ }^{5}$ Gray spheres (only shown on left side) represent sites at which DBS elicited hypomania. ${ }^{21,23,24}$ (RN = red nucleus; STN = subthalamic nucleus; SNr = substantia nigra; VTA = ventral tegmental area; PAG = periaquaeductal gray; PT = pyramidal tract; $\mathrm{Ih}=$ lateral hypothalamus; imMFB = inferomedial medial forebrain bundle; $f x=$ fornix; sIMFB = superolateral medial forebrain bundle; viii $=$ third ventricle; $\mathrm{mtt}=$ mamillo-thalamic tract; $\mathrm{ATN}=$ anterior thalamic nucleus; DMT $=$ dorsomedial thalamus; rc Hipp $=$ retro-commissural hippocampus; NAC = nucleus accumbens; ALIC = anterior limb of internal capsule; SCG = subgenual cingulate gyrus; ATR = anterior thalamic radiation; $\mathrm{PFC}=$ prefrontal cortex.)

stimulation response of the prefronto-mesencephalic descending fibers that enter the VTA. ${ }^{7,8}$ This response could lead to a combined modulation (reduction of physic activity, induction of tonic activity) of the VTA, the prefrontal cortex (PFC), and OFC (and the ATR), and likely to a dopamine release in the ventral striatum. ${ }^{19}$

Patients with MD react to slMFB DBS with an acute increase of appetitive motivation (goal directed behavior) owing to an improved prediction of distant reward. ${ }^{7,8}$ Reward prediction behavior was shown in rodents to be linked to the level of free synaptic dopamine in the ventral striatum. $^{20}$ In sIMFB DBS, a stimulation of fibers that descend into the VTA might (as suggested for rituals) transfer the function of dopaminergic neurons from phasic high-frequency oscillation into a tonic activity and might exert anti-OCD effects over this modulation of the reward system (Figure 4). The authors believe that medial STN DBS in $\mathrm{OCD}^{21}$ works over the same mode of action by modulating the nearby slMFB. ${ }^{22}$ Clinically, hypomania-which might be conceptualized as a pathological activation of the reward system-can be seen during DBS for OCD in a variety of targets $^{21,23-25}$ (Figure 3). In OCD it has been shown that
DBS to the reward system (nucleus accumbens) increases striatal dopamine release, ${ }^{26}$ which under certain circumstances might be a likely correlate of hypomania. In STN DBS, medial electrode positions were assumed to induce hypomania by co-activation of the sIMFB. ${ }^{22}$ However, results from rodent research are somewhat contradictory. According to some authors, DBS of the MFB does not lead to an increase in dopamine release in the striatum (in models of depression), ${ }^{27}$ whereas our own rodent research shows a crucial role of this very dopamine transmission for the mode of action of chronic MFB DBS. ${ }^{19,28,29}$

It is worth noting that there is a significant comorbidity of OCD and major depression. Most consistently in OCD, a neuroimaging finding of a relative hypermetabolism of the orbitofrontal cortex and anterior cingulate cortex is reported. This hypermetabolism can be reduced by both pharmacological and/or behavioral therapy and also by DBS. Reduction of this hypermetabolism might be thought to be the imaging correlate of clinical improvement, ${ }^{15}$ although the mechanism of this has not readily been explained.

The stimulation approaches in different target regions are characteristic for high stimulation amplitudes. This has 


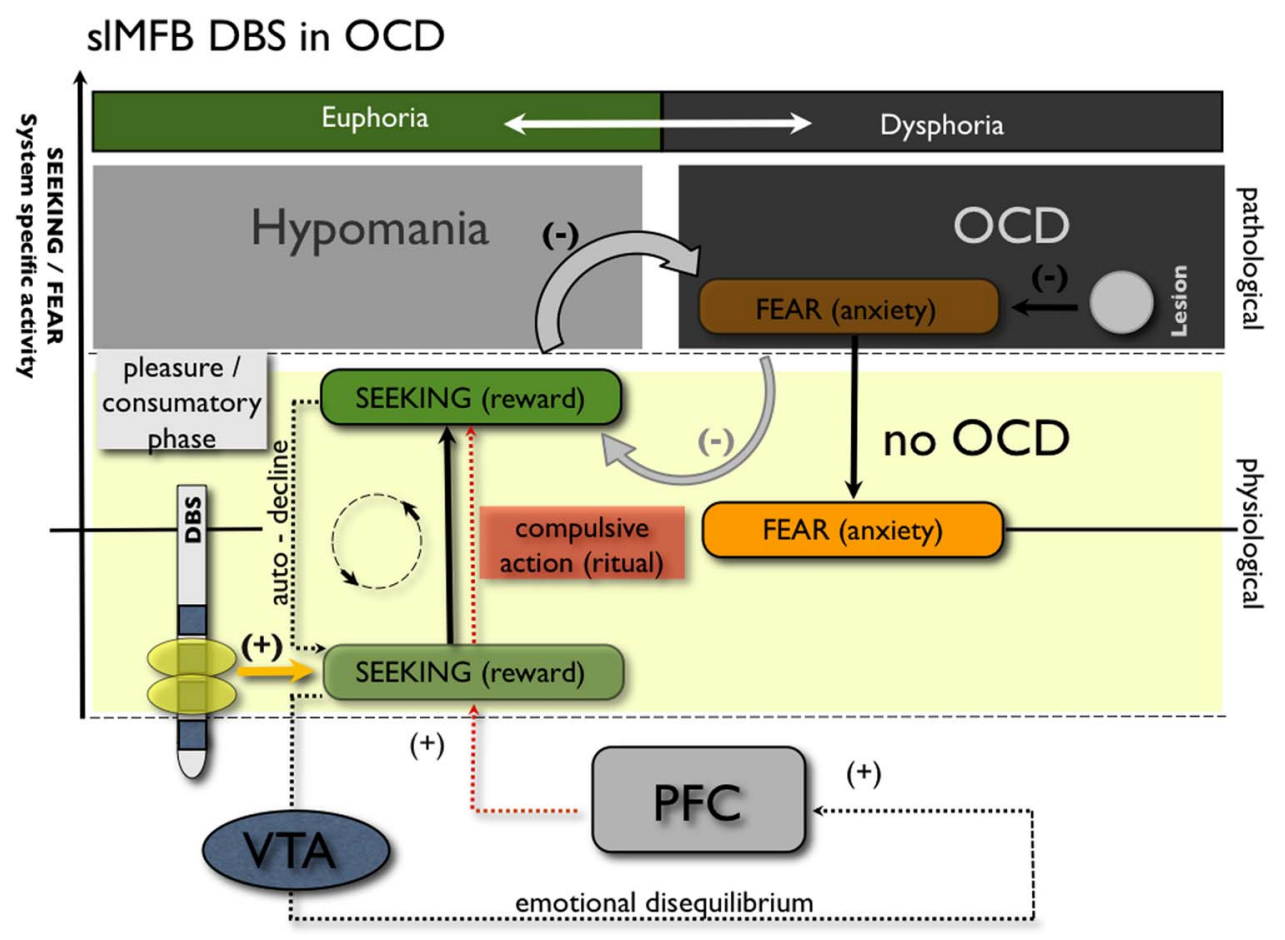

FIGURE 4. Conceptual mode of action of SIMFB DBS for OCD. In this proposed OCD framework, the reward system is incapable of compensating for high activity in the FEAR system (promoting anxiety, anatomically realized in the ATR; Figure 3). This network can be perturbed at different points. For example, a lesion (eg, to the anterior limb of the internal capsule) directly reduces high activity in the FEAR system. Repetitive compulsions (rituals) transfer phasic activity in the SEEKING system into tonic activity. This, though transiently, reduces fear and anxiety. SIMFB DBS introduces tonic activity into the SEEKING (reward) system utilizing the same physiological mechanism. (Capitalization refers to affective neuroscience context in this interpretation. ${ }^{10,11}$ )

been criticized in the literature and was used as an indicator for a suboptimal target region. ${ }^{14}$ Stimulation studies looking at the depression circuitry (which includes most of the key regions for the OCD symptomatology) found that a better target region could be the slMFB, just where it exits the VTA. ${ }^{11}$ The putative mode of action as proposed here adds important regions to the previously proposed deregulated CSTC network and stresses its interaction with the reward circuitry (especially MFB and VTA).

Certain limitations with respect to our study have to be noted. We have not used field simulation techniques to evaluate the volume of activated tissue in our approach. This evaluation is the focus of a separate study. We have not seen adverse events in our patients, but owing to the case report character of this work, there was no control of vigilance. It could be pure luck that these patients did not show any adverse events. In our previous work, we have described a unilateral hemorrhage in the subthalamic target region. ${ }^{7}$ However, in overall 27 bilateral implantations, we have so far only seen this singular bleeding episode. Not everything is said, obviously, on the complication rate of slMFB DBS. Likely, the complication rate will be very similar to the one of the depressed population. This also is focus of ongoing research while exploring the slMFB as a target region.

\section{Conclusions}

We demonstrated in this uncontrolled case series study that continuous bilateral stimulation of the sIMFB led to significant clinical improvement of OCD. Our ongoing research focuses first on the time scale on which the antiOCD effects in this target region occur. Second, we are interested in the role of the reward system in the genesis and the treatment of OCD. Anti-OCD effects occurred in our patients with very low stimulation amplitudes. This, together with the fact that the slMFB is a target region that combines imaging information with electrophysiology and an intra-operatively testable functional environment-and thus holds many features of typical movement disorder target regions (like the subthalamic nucleus for Parkinson's disease)-could make the slMFB an interesting new target region for therapy refractory OCD. We have offered a new and alternative mode of action for this stimulation approach and a scientific framework that shows one path for future research with a focus on the reward circuitry. Controlled studies are needed to further investigate sIMFB's efficacy for the treatment of OCD. 


\section{Disclosures}

Dr. Coenen reports grants and other from Boston Scientific, USA; grants from Medtronic, USA; personal fees from Boston Scientific, USA; personal fees from Medtronic, USA; grants from BrainLinks/Brain Tools cluster of excellence, outside the submitted work. Dr. Freyer and Tebartz van Elst Ludger have nothing to disclose. Dr. Reinacher reports grants, personal fees, and non-financial support from Medtronic Neuromodulation Branch, Europe; grants and personal fees from Boston Scientific, USA; grants and personal fees from German Federal Ministry for Economic Affairs and Energy, outside the submitted work; and travel support and honoraria for talks for Boston Scientific (USA). Dr. Schlaepfer reports partial funding by Medtronic Inc. for three IIT's on which I am PI (in the last 2 years). Horst Urbach has nothing to disclose. Prof. Ulrich Voderholzer has nothing to disclose. Dr. Goll reports grants from Otsuka Pharma, and grants from Boston Scientific, outside the submitted work.

\section{REFERENCES:}

1. Koran LM, Hanna GL, Hollander E, Nestadt G, Simpson HB, American Psychiatric Association. Practice guideline for the treatment of patients with obsessive-compulsive disorder. $\mathrm{Am} \mathrm{J}$ Psychiatry. 2007; 164(7 Suppl): 5-53.

2. Nuttin B, Cosyns P, Demeulemeester H, Gybels J, Meyerson B. Electrical stimulation in anterior limbs of internal capsules in patients with obsessive-compulsive disorder. Lancet. 1999; 354(9189): 1526.

3. Bourne SK, Eckhardt CA, Sheth SA, Eskandar EN. Mechanisms of deep brain stimulation for obsessive compulsive disorder: effects upon cells and circuits. Front Integr Neurosci. 2012; 6: 29.

4. Greenberg BD, Rauch SL, Haber SN. Invasive circuitry-based neurotherapeutics: stereotactic ablation and deep brain stimulation for OCD. Neuropsychopharmacology. 2010; 35(1): 317-336.

5. Kisely S, Hall K, Siskind D, Frater J, Olson S, Crompton D. Deep brain stimulation for obsessive-compulsive disorder: a systematic review and meta-analysis. Psychol Med. 2014; 44(16): 3533-3542.

6. Ahmari SE, Dougherty DD. Dissecting OCD circuits: from animal models to targeted treatments. Depress Anxiety. 2015; 32(8): 550-562.

7. Schlaepfer TE, Bewernick BH, Kayser S, Madler B, Coenen VA. Rapid effects of deep brain stimulation for treatment-resistant major depression. Biol Psychiatry. 2013; 73(12): 1204-1212.

8. Schlaepfer TE, Bewernick BH, Kayser S, Hurlemann R, Coenen VA. Deep brain stimulation of the human reward system for major depression-rationale, outcomes and outlook. Neuropsychopharmacology. 2014; 39(6): 1303-1314.

9. Döbrössy MD, Furlanetti LL, Coenen VA. Electrical stimulation of the medial forebrain bundle in pre-clinical studies of psychiatric disorders. Neurosci Biobehav Rev. 2015; 49: 32-42.

10. Coenen VA, Panksepp J, Hurwitz TA, Urbach H, Madler B. Human medial forebrain bundle (MFB) and anterior thalamic radiation (ATR): imaging of two major subcortical pathways and the dynamic balance of opposite affects in understanding depression. J Neuropsychiatry Clin Neurosci. 2012; 24(2): 223-236.

11. Coenen VA, Schlaepfer TE, Maedler B, Panksepp J. Cross-species affective functions of the medial forebrain bundle-implications for the treatment of affective pain and depression in humans. Neurosci Biobehav Rev. 2011; 35(9): 1971-1981.
12. Zaitsev M, Hennig J, Speck O. Point spread function mapping with parallel imaging techniques and high acceleration factors: fast, robust, and flexible method for echo-planar imaging distortion correction. Magn Reson Med. 2004; 52(5): 1156-1166.

13. Ahmari SE, Spellman T, Douglass NL, et al. Repeated corticostriatal stimulation generates persistent OCD-like behavior. Science. 2013; 340(6137): 1234-1239.

14. Lipsman N, Neimat JS, Lozano AM. Deep brain stimulation for treatment-refractory obsessive-compulsive disorder: the search for a valid target. Neurosurgery. 2007; 61(1): 1-11; discussion 11-13.

15. Van Laere K, Nuttin B, Gabriels L, et al. Metabolic imaging of anterior capsular stimulation in refractory obsessive-compulsive disorder: a key role for the subgenual anterior cingulate and ventral striatum. J Nucl Med. 2006; 47(5): 740-747.

16. Chaudhury D, Walsh JJ, Friedman AK, et al. Rapid regulation of depression-related behaviours by control of midbrain dopamine neurons. Nature. 2013; 493(7433): 532-536.

17. Hurwitz TA, Mandat T, Forster B, Honey C. Tract identification by novel MRI signal changes following stereotactic anterior capsulotomy. Stereotact Funct Neurosurg. 2006; 84(5-6): 228-235.

18. Schoene-Bake JC, Parpaley Y, Weber B, Panksepp J, Hurwitz TA, Coenen VA. Tractographic analysis of historical lesion surgery for depression. Neuropsychopharmacology. 2010; 35(13): 2553-2563.

19. Furlanetti LL, Coenen VA, Dobrossy MD. Ventral tegmental area dopaminergic lesion-induced depressive phenotype in the rat is reversed by deep brain stimulation of the medial forebrain bundle. Beha Brain Res. 2016; 299: 132-140.

20. Howe MW, Tierney PL, Sandberg SG, Phillips PE, Graybiel AM. Prolonged dopamine signalling in striatum signals proximity and value of distant rewards. Nature. 2013; 500(7464): 575-579.

21. Mallet L, Polosan M, Jaafari N, et al. Subthalamic nucleus stimulation in severe obsessive-compulsive disorder. $N$ Engl J Med. 2008; 359(20): 2121-2134.

22. Coenen VA, Honey CR, Hurwitz T, et al. Medial forebrain bundle stimulation as a pathophysiological mechanism for hypomania in subthalamic nucleus deep brain stimulation for Parkinson's disease. Neurosurgery. 2009; 64(6): 1106-1114; discussion 1114-1115.

23. Tsai HC, Chen SY, Tsai ST, Hung HY, Chang CH. Hypomania following bilateral ventral capsule stimulation in a patient with refractory obsessive-compulsive disorder. Biol Psychiatry. 2010; 68(2): e7-e8.

24. Chang CH, Chen SY, Hsiao YL, Tsai ST, Tsai HC. Hypomania with hypersexuality following bilateral anterior limb stimulation in obsessivecompulsive disorder. J Neurosurg. 2010; 112(6): 1299-1300.

25. Widge AS, Licon E, Zorowitz S, et al. Predictors of hypomania during ventral capsule/ventral striatum deep brain stimulation. J Neuropsychiatry Clin Neurosci. 2016; 28(1): 38-44.

26. Figee M, de Koning P, Klaassen S, et al. Deep brain stimulation induces striatal dopamine release in obsessive-compulsive disorder. Biol Psychiatry. 2014; 75(8): 647-652.

27. Bregman T, Reznikov R, Diwan M, et al. Antidepressant-like effects of medial forebrain bundle deep brain stimulation in rats are not associated with accumbens dopamine release. Brain Stimulation. 2015; 8(4): 708-713.

28. Furlanetti LL, Coenen VA, Aranda IA, Dobrossy MD. Chronic deep brain stimulation of the medial forebrain bundle reverses depressivelike behavior in a hemiparkinsonian rodent model. Exp Brain Res. 2015; 233(11): 3073-3085.

29. Furlanetti LL, Dobrossy MD, Aranda IA, Coenen VA. Feasibility and safety of continuous and chronic bilateral deep brain stimulation of the medial forebrain bundle in the naive Sprague-Dawley rat. Behavioural neurology. 2015; 2015: 256196.

30. Farris SG, McLean CP, Van Meter PE, Simpson HB, Foa EB. Treatment response, symptom remission, and wellness in obsessivecompulsive disorder. J Clin Psychiatry. 2013; 74(7): 685-690. 